\title{
Neither backward masking of T2 nor task switching is necessary for the attentional blink
}

\author{
Ali Jannati • Thomas M. Spalek • Vincent Di Lollo
}

Published online: 16 November 2010

(C) Psychonomic Society, Inc. 2010

\begin{abstract}
Identification of the second of two targets (T1, T2, inserted in a stream of distractors) is impaired when presented within $500 \mathrm{~ms}$ after the first (attentional blink, AB). Barring a T1-T2 task-switch, it is thought that $\mathrm{T} 2$ must be backward-masked to obtain an $\mathrm{AB}$ (Giesbrecht \& Di Lollo, Journal of Experimental Psychology: Human Perception and Performance, 24, 14541466, 1998). We tested the hypothesis that Giesbrecht \& Di Lollo's findings were vitiated by ceiling constraints arising from either response scale (experiment 1 ) or data limitations (experiment 2). In experiment 1, digitdistractors were replaced with pseudoletters to increase task difficulty, bringing performance below ceiling. An $A B$ occurred without backward masking of $T 2$. In experiment 2 , a ceiling-free procedure estimated the number of noise dots needed for $80 \%$ T2 identification. $A n A B$ was revealed: fewer noise dots were required during the $\mathrm{AB}$ period than outside it. Both outcomes confirm that an $\mathrm{AB}$ can be obtained without either masking of $\mathrm{T} 2$ or task switching.
\end{abstract}

\section{Keywords Attentional blink · Masking · Task switching}

When two targets (T1 and T2) are inserted in a stream of distractors presented in rapid serial visual presentation (RSVP), identification accuracy is nearly perfect for T1 but is dramatically reduced for T2 (Raymond, Shapiro, \& Arnell, 1992). This phenomenon, called the attentional

A. Jannati $(\varangle) \cdot$ T. M. Spalek $\cdot$ V. Di Lollo

Department of Psychology, Simon Fraser University,

8888 University Drive,

Burnaby, BC, Canada V5A 1S6

e-mail: ali_jannati@alumni.sfu.ca blink (AB), is most evident when $\mathrm{T} 2$ is presented with a temporal lag of $100-500 \mathrm{~ms}$ after T1.

The underlying mechanisms of this second-target deficit are currently a matter of debate (for reviews see Dux \& Marois, 2009, and Martens \& Wyble, 2010). However, there is general agreement that in order to obtain an $A B$ it is necessary that $\mathrm{T} 2$ be backward-masked. This is why in the $\mathrm{AB}$ literature $\mathrm{T} 2$ is typically followed by at least one other item that acts as a backward mask ${ }^{1}$. This practice is largely based on Giesbrecht and Di Lollo's (1998) finding that the $\mathrm{AB}$ is not in evidence unless $\mathrm{T} 2$ is followed by a trailing item. Here we show that backward masking of T2 is not essential for the $\mathrm{AB}$, and that Giesbrecht and Di Lollo's findings were vitiated by constraints arising from the experimental design.

Giesbrecht and Di Lollo (1998) studied the role of T2 masking in two main experiments. In experiment 1 , observers were required to identify two letter targets inserted in an RSVP stream of digit distractors. A significant $\mathrm{AB}$ was obtained when $\mathrm{T} 2$ was followed by a digit that functioned as a backward mask, but not when $\mathrm{T} 2$ was the last item in the RSVP stream. The authors noted, however, that since performance in the no-mask condition was near perfect, the absence of an $\mathrm{AB}$ might have been due to a ceiling constraint imposed by the $100 \%$ limit of the response scale.

To rule out this possibility, Giesbrecht and Di Lollo (1998) implemented a condition in experiment 2 in which accuracy of T2 identification was brought below ceiling by embedding the $\mathrm{T} 2$ letter in an aggregate of random dots that

\footnotetext{
${ }^{1}$ An exception to this rule is when a task switch occurs between T1 and $\mathrm{T} 2$. In that case, a small but significant $\mathrm{AB}$ has been reported (Kawahara, Di Lollo, \& Enns, 2001; Kawahara, Zuvic, Enns, \& Di Lollo, 2003). The task switch is said to delay the processing of T2 during which the $\mathrm{T} 2$ representation decays beyond recognition.
} 
lowered the level of performance to approximately $80 \%$ correct responses. Yet, no $\mathrm{AB}$ was in evidence. On the strength of these findings, Giesbrecht and Di Lollo concluded that backward masking of $\mathrm{T} 2$ was essential for the $\mathrm{AB}$, and that the absence of an $\mathrm{AB}$ in experiment 1 was not due to ceiling constraints.

However, there is a possibility that the impoverishment of T2 in Giesbrecht and Di Lollo's (1998) experiment 2 might have created a new ceiling arising not from the upper limit of the response scale but from what Norman and Bobrow (1975) referred to as data limitation. Namely, the level of impoverishment might have been such that the T2 letter could not be identified on more than about $80 \%$ of the trials even when presented at the longest lag of $700 \mathrm{~ms}$, which is beyond the period of the AB. Were it not for such a data-limitation ceiling, an $\mathrm{AB}$ might have been in evidence with no backward masking of $\mathrm{T} 2$.

To summarize, it is possible that the absence of an $\mathrm{AB}$ in Giesbrecht and Di Lollo's (1998) study might have been due to two different forms of constraint: the upper limit of the response scale in experiment 1 , and data limitation in experiment 2. The present work examined both forms of constraint. First, we examined the possibility that in Giesbrecht and Di Lollo's experiment 1, when T2 was neither impoverished nor followed by a mask, performance was constrained by the $100 \%$ limit because the task was too easy. The present experiment 1 was designed to lower the identification accuracy for $\mathrm{T} 2$ below $100 \%$ by using pseudoletters instead of digit distractors. The ensuing increased target-distractor similarity was expected to lead to a corresponding impairment in $\mathrm{T} 2$ identification. The intent was to bring T2 performance below the $100 \%$ ceiling (Chun \& Potter, 1995; Visser, Bischof, \& Di Lollo, 2004) and thus allow any latent $\mathrm{AB}$ to be revealed.

Next, we examined the possibility that in Giesbrecht and Di Lollo's (1998) study when T2 was impoverished by randomdot noise, the $\mathrm{AB}$ was not in evidence because of data limitation. This was done in experiment 2 by using a dynamic threshold-tracking method that is not constrained either by response-scale limits or by data limitations (PEST; Parameter Estimation by Sequential Testing, Taylor \& Creelman, 1967). PEST is not constrained by either ceiling or floor considerations because if either constraint is present, PEST cannot converge on the criterial level. To the extent that failure to find an $\mathrm{AB}$ was due to ceiling constraints, an $\mathrm{AB}$ should be in evidence when those constraints are removed.

\section{Experiment 1}

The objective of experiment 1 was to lower T2 performance below $100 \%$ by increasing the difficulty of the task relative to that in the study of Giesbrecht and Di Lollo (1998). As

was the case in the latter, no mask was presented after T2. Task difficulty was increased by increasing the similarity of the distractors to the letter target. This was done by replacing the digit distractors with pseudoletters. If an $\mathrm{AB}$ were found with pseudoletter distractors, it would mean that the $\mathrm{AB}$ can indeed occur with no backward masking of $\mathrm{T} 2$. It would also mean that performance in the easier task used by Giesbrecht and Di Lollo was probably constrained by the $100 \%$ limit of the response scale.

Methods

\section{Observers}

Twenty-seven undergraduate students participated for course credit. All reported normal or corrected-to-normal vision and were naive to the purpose of the experiment.

\section{Apparatus and stimuli}

The experiment was run in a dimly lit room. Observers sat at a distance of approximately $60 \mathrm{~cm}$ from a NEC AccuSync 120 computer monitor refreshed at a rate of $140 \mathrm{~Hz}$. They viewed an RSVP stream that included black pseudoletter distractors and two target letters (all 48-point Geneva font, height $1.0^{\circ}$ ) in the centre of the screen against a white background. Each item remained on the screen for $50 \mathrm{~ms}$, and was followed by a 50$\mathrm{ms}$ inter-stimulus interval (ISI) during which the screen remained blank, yielding a presentation rate of ten items/s. The RSVP stream contained a variable number of pseudoletter distractors and two letter targets (T1 and T2) selected randomly from the English alphabet, except I, O, Q, and Z. The number of distractors preceding $\mathrm{T} 1$ was determined randomly on each trial and varied between five and ten, inclusive. On any given trial, the distractors were drawn from the set of pseudoletters illustrated in Fig. 1, with the constraint that the selected pseudoletter was not one of the two preceding items.

T2 was presented at one of three lags after T1: 100, 300, or $900 \mathrm{~ms}$, i.e., lags 1, 3, and 9, respectively. The lag was selected randomly on each trial, with the constraint that there was an equal number of trials per lag. Pseudoletter distractors continued to be presented during the inter-target lag. The RSVP stream ended with a 7-ms presentation of T2. The sequence of events on any given trial is illustrated in the left-hand panel of Fig. 2. In the present context, the

Fig. 1 Pseudoletters used in experiments 1 and 2

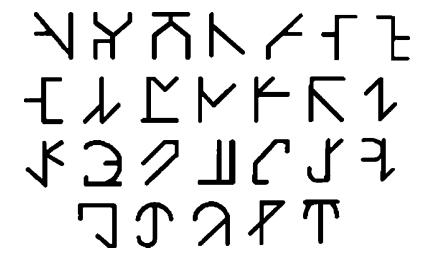




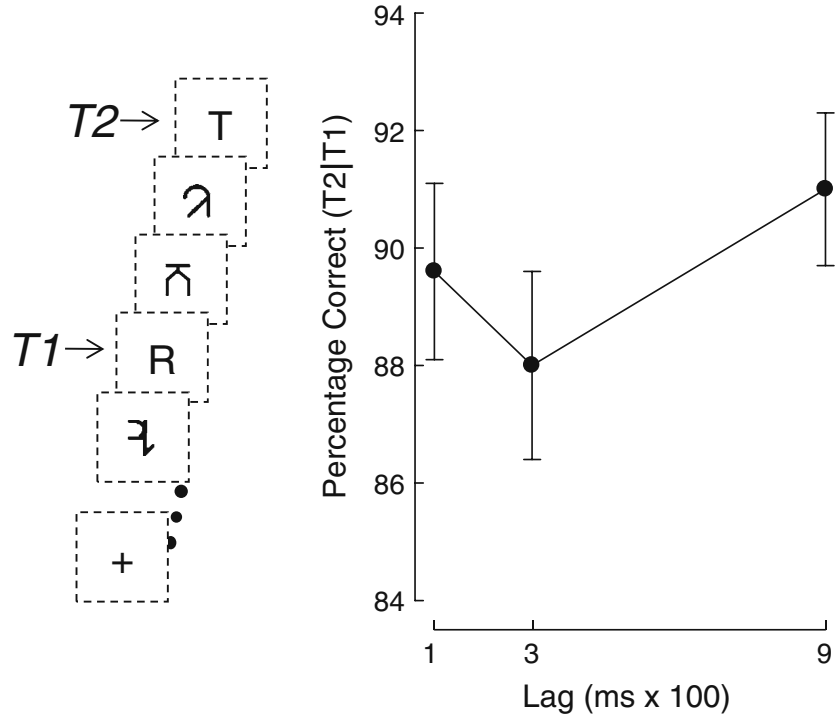

Fig. 2 Left-hand panel: display sequence in experiment 1. Illustrated is an inter-target lag of $300 \mathrm{~ms}$ (lag 3). Right-hand panel: Mean percentage of correct $\mathrm{T} 2$ responses at each lag given that $\mathrm{T} 1$ had been reported correctly $(\mathrm{T} 2 \mid \mathrm{T} 1) . T 1=$ first target; $T 2=$ second target

$\mathrm{AB}$ was defined as the difference between lags 3 and 9. Lag 1 was included for consistency with previous $\mathrm{AB}$ studies.

\section{Design and procedure}

At the beginning of each trial, a small fixation cross was presented in the center of the screen. Observers initiated each trial by pressing the space bar. They were instructed to ignore the distractors and to report the identity of $\mathrm{T} 1$ and $\mathrm{T} 2$, one at a time at their leisure, by pressing the corresponding keys on the keyboard in response to prompts on the screen that were displayed approximately $10^{\circ}$ above the RSVP stream. This substantial spatial separation obviated any backward masking of $\mathrm{T} 2$. Observers were also instructed that they could report the targets in either order, and that they could guess if unsure. The experiment began with 10 practice trials, which were followed by 300 trials, 100 for each of the three lags.

\section{Results and discussion}

In this and the next experiment, only those trials in which T1 was identified correctly were included for analysis. This procedure is commonly adopted in $\mathrm{AB}$ experiments on the grounds that, on trials in which $\mathrm{T} 1$ is identified incorrectly, the source of the error is unknown, and thus its effect on T2 processing cannot be estimated. Collapsed across lags, the percentage of correct $\mathrm{T} 1$ responses was 80.1. The $\mathrm{T} 2$ results, illustrated in Fig. 2, were analyzed in a one-way within-subject analysis of variance (ANOVA) with lag as the factor at three levels (lag 1, lag 3, and lag 9). The analysis revealed a marginally significant effect of Lag, $F(2,52)=2.99, p=0.059, \mathrm{~ms} E=25.26$. The quadratic component was significant, $F(1,26)=5.23, p=0.031$, $\mathrm{ms} E=23.81$. A planned paired $t$ test between lags 3 and 9 revealed a significant $\mathrm{AB}, \mathrm{t}(26)=2.75, p=0.011$.

As illustrated in Fig. 2, the overall level of T2 performance was close to ceiling. However, increasing the target-distractor similarity by using pseudoletters was successful in revealing a significant $\mathrm{AB}$, even when $\mathrm{T} 2$ was not followed by a mask. This result demonstrates that the absence of a significant $\mathrm{AB}$ in the corresponding experiment by Giesbrecht and Di Lollo (1998) might have been due to a ceiling constraint imposed by the response scale. The essential point is that an $\mathrm{AB}$ can be obtained without backward masking of $\mathrm{T} 2$ and without a task switch between the two targets.

\section{Experiment 2}

Experiment 2 was designed to examine the possibility, outlined in the introduction, that the absence of an $\mathrm{AB}$ in Giesbrecht and Di Lollo's (1998) experiment 2 in which T2 was degraded with random-dot noise might have stemmed from a data-limitation ceiling. To avoid such a ceiling, we used a method which, as noted above, is free from ceiling constraints. Instead of using accuracy as a response measure, we determined the number of noise dots that were required to identify $\mathrm{T} 2$ on about $80 \%$ of the trials. We reasoned that, to the extent that the processing of $\mathrm{T} 2$ is impaired during the period of the $\mathrm{AB}$, a lower level of degradation (fewer noise dots) would be needed at lag 3 than at lag 9 to maintain the criterial level of performance.

The number of random dots presented simultaneously with T2 was varied dynamically by PEST at each lag, separately for each observer. PEST was set to converge to a level of $80 \%$ correct $\mathrm{T} 2$ identifications, given that $\mathrm{T} 1 \mathrm{had}$ been identified correctly (T2|T1). On the hypothesis that backward masking is necessary for the $\mathrm{AB}$, the number of noise dots required to reach the $80 \%$ criterion $\left(\mathrm{DOT}_{\mathrm{C}}\right)$ should be invariant with lag when T2 is not followed by a trailing mask. In contrast, a smaller $\mathrm{DOT}_{\mathrm{C}}$ at lag 3 than at lag 9 would indicate that T2 identification at lag 3 is relatively impaired, consistent with the conclusion that an $\mathrm{AB}$ can occur with no backward masking of $\mathrm{T} 2$.

Methods

Observers

Eighteen undergraduate students participated for course credit. They were drawn from the same population as 
experiment 1. None had participated in the previous experiment.

\section{Apparatus and stimuli}

Apparatus and stimuli were the same as in experiment 1 except for the following. An aggregate of noise dots was overlaid on T2. Each dot consisted of a small black patch $\left(2 \times 2\right.$ pixels, $0.1^{\circ}$ side $)$. The location of each dot was chosen randomly within an imaginary square (height $0.9^{\circ}$ ) containing the $\mathrm{T} 2$ letter. The number of dots was varied by PEST, as described below.

\section{Design and procedure}

Design and procedures were the same as in experiment 1, with the following exceptions. The exposure duration of T2 was $50 \mathrm{~ms}$. The dependent measure was the value of

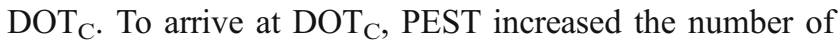
noise dots when the task was too easy (T2|T1 over the criterial level), and reduced it when the task was too hard. A Wald (1947) sequential likelihood-ratio test determined whether the immediately preceding run of responses was above or below criterial level. The Wald routine was called only on trials in which $\mathrm{T} 1$ had been identified correctly. The assumption was made that the criterial level of performance had been approximated after four reversals in the direction of adjustment of the number of noise dots. The final score $\left(\mathrm{DOT}_{\mathrm{C}}\right)$ was the mean number of dots over the 16 trials following the fourth reversal. Thus, $\mathrm{DOT}_{\mathrm{C}}$ represented the level of $\mathrm{T} 2$ degradation required to achieve the criterial level. The observers were instructed to report the two targets in any order, guessing if unsure. For each observer, three concurrent and randomly intermixed PEST sequences were run, one for each lag.

\section{Results and discussion}

Collapsed across lags, the percentage of correct T1 responses was 90.3. The percentages of correct T2|T1 responses were 78.9, 78.5, and 81.3 for lags 1, 3, and 9, respectively. The values of $\mathrm{DOT}_{\mathrm{C}}$, illustrated in Fig. 3, were analyzed in a one-way within-subject ANOVA with lag as the factor at three levels (lags 1, 3, and 9). The analysis revealed a marginally significant effect of lag, $F(2,34)=$ 3.22, $p=0.052, \mathrm{~ms} E=26.77$. The quadratic component was significant, $F(1,17)=5.88, p=.027, \mathrm{~ms} E=26.60$. A planned paired $t$ test between lags 3 and 9 revealed a significant $\mathrm{AB}, \mathrm{t}(17)=2.48, p=0.024$.

The results of experiment 2 are unambiguous: fewer noise dots could be tolerated at lag 3 than at lag 9 to achieve the criterial level of about $80 \%$ correct $\mathrm{T} 2 \mid \mathrm{T} 1$ identifications. Clearly, an $\mathrm{AB}$ can be obtained in the

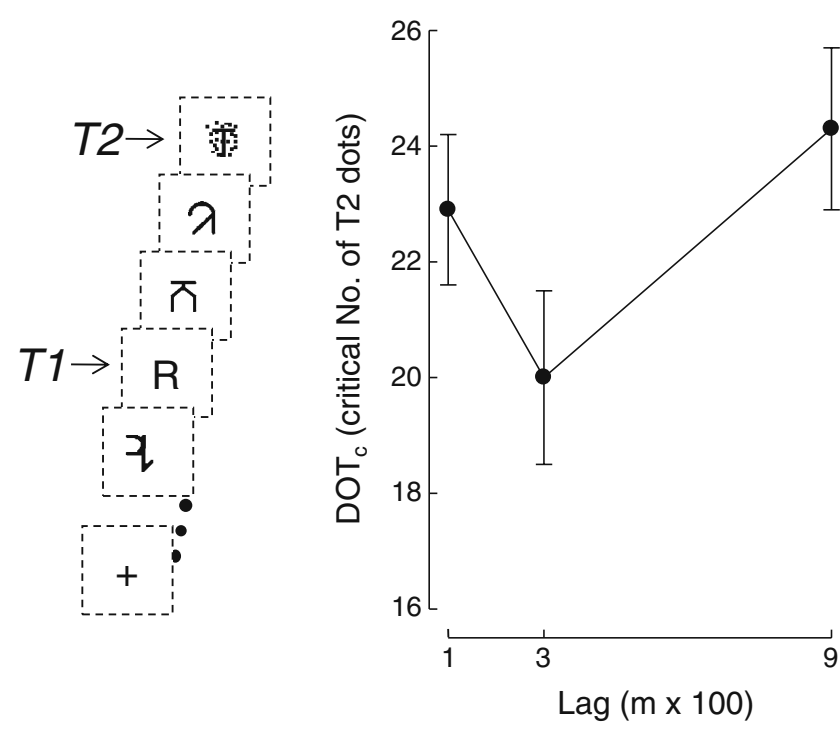

Fig. 3 Left-hand panel: display sequence in experiment 2. Illustrated is an inter-target lag of $300 \mathrm{~ms}$ (lag 3 ). The number of noise dots overlaid on $\mathrm{T} 2$ was varied dynamically to converge on $80 \%$ correct $\mathrm{T} 2$ identifications $\left(\mathrm{DOT}_{\mathrm{C}}\right)$. Right-hand panel: Mean $\mathrm{DOT}_{\mathrm{C}}$ scores averaged across observers at each lag. $T 1=$ first target; $T 2=$ second target

absence of any backward masking of T2. Furthermore, the present results indicate that a data-limitation ceiling was probably responsible for the absence of an AB in the study of Giesbrecht \& Di Lollo, (1998, experiment 2), in which T2 was degraded by a fixed number of noise dots.

\section{General discussion}

It is generally agreed that, unless there is a task switch between the two targets, T2 must be backward-masked in order to obtain an AB (Giesbrecht \& Di Lollo, 1998; Kawahara, Di Lollo, \& Enns, 2001; Kawahara, Zuvic, Enns, \& Di Lollo, 2003; Vogel \& Luck, 2002). Here we show that an $\mathrm{AB}$ can occur without backward masking of $\mathrm{T} 2$ even in the absence of task switching.

In experiment 1, we tested the hypothesis that Giesbrecht and Di Lollo (1998, experiment 1) failed to find an AB when T2 was not backward-masked because performance was constrained by a response-scale ceiling. We used pseudoletter distractors instead of digits to increase the difficulty of the task thus bringing performance below ceiling. We found a small but significant $\mathrm{AB}$ even though T2 was not backward-masked.

Experiment 2 examined the possibility that a different type of ceiling, arising from data limitations, might have prevented an $\mathrm{AB}$ in the study of Giesbrecht and Di Lollo (1998, experiment 2). Such a ceiling was averted by using a dynamic threshold tracking procedure in which T2 was not backward-masked but was overlaid by a variable number of noise dots. The procedure determined the number of noise 
dots that could be tolerated to converge on about $80 \%$ correct $\mathrm{T} 2$ identifications at each lag. The results revealed a significant $\mathrm{AB}$ : fewer noise dots were required at lag 3 than at either lags 1 or 9 , strongly suggesting that the absence of an $\mathrm{AB}$ in Giesbrecht \& Di Lollo's study was due to a datalimitation ceiling. The results of both experiments confirmed that an $\mathrm{AB}$ can be obtained without backward masking of T2 and without task switching.

\section{$\mathrm{T} 2$ deterioration during the $\mathrm{AB}$}

It is known that the processing of T2 is delayed during the period of the AB (Jolicœur \& Dell'Acqua, 1998; Visser, 2007; Vogel \& Luck, 2002). During this delay, the T2 representation is held to be vulnerable to decay and to backward masking, thus giving rise to the $\mathrm{AB}$ (Chun \& Potter, 1995; Kawahara et al., 2001, 2003). In the present work, backward masking was obviously not a factor, leaving decay as the most likely cause of the AB.

The idea of a decaying T2 representation is encompassed naturally within two-stage (bottleneck) models in which some critical stage of consolidation of the T2 representation is said to be postponed (Chun \& Potter, 1995; Jolicœur \& Dell'Acqua, 1998). While in a non-consolidated state, the T2 representation decays over time and is vulnerable to masking by trailing items. Thus, a trailing mask is not essential for the $A B$, although it probably increases its magnitude. In contrast, input-control models ascribe the $\mathrm{AB}$ to interference arising not from the requirement to process $\mathrm{T} 1$ but to the presence of intervening distractors that disrupt or suppress an input filter (Di Lollo, Kawahara, Ghorashi, \& Enns, 2005; Olivers \& Meeter, 2008). As presently stated, input-control theories stipulate that masking of $\mathrm{T} 2$ is essential for the $\mathrm{AB}$, and do not specify any mechanisms that could account for the presence of an $\mathrm{AB}$ when $\mathrm{T} 2$ is not masked. Thus, input-control models would need to be modified in order to encompass the present results. A detailed comparison between limited-capacity and inputcontrol theories of the $\mathrm{AB}$ is beyond the scope of the present paper. A recent debate on this issue can be found in papers by Dux, Asplund, and Marois (2008), who advocated a resource-depletion account (but see response by Olivers, Spalek, Kawahara, \& Di Lollo, 2009), and by Dell'Acqua, Jolicœur, Luria, and Pluchino (2009) who also advocated a capacity-limitation account (but see response by Olivers, Hulleman, Spalek, Kawahara, \& Di Lollo, 2010).

It is perhaps worth noting that, in earlier studies, an $\mathrm{AB}$ attributable to decay has been found only when the T2 stimulus was severely degraded (Kawahara et al., 2001). This is because, when so degraded, the T2 representation decays to illegibility before the processing delay is over, thus giving rise to an $\mathrm{AB}$. In contrast, when the T2 stimulus is not degraded, it is more likely to remain legible until after the processing delay is over, thus averting an $\mathrm{AB}$.

Decay of the $\mathrm{T} 2$ representation during the period of delay is clearly not the only determinant of the AB. Rather, in conventional studies, decay and backward masking are likely to work in conjunction with one another. A direct comparison between the $\mathrm{DOT}_{\mathrm{C}}$ measure used in experiment 2 and the accuracy measure used in conventional $\mathrm{AB}$ studies is obviously not feasible. At a first approximation, the magnitude of the $\mathrm{AB}$ can be estimated as 1 minus the ratio of the dependent measures at lags 3 and 9. In Giesbrecht \& Di Lollo's study (1998, Fig. 2a), in which the $\mathrm{T} 2$ representation was weakened by both masking and decay, that value was .33 . The corresponding value in the present experiment 2 , in which the $\mathrm{T} 2$ representation was weakened only by decay, the value was .18. Regardless of specifics, the main contribution of the present work lies in the decoupling of the decay and the masking components of the $\mathrm{AB}$.

Author Note This work was supported by a British Columbia Pacific Century Graduate Scholarship to AJ, by a Canadian Foundation for Innovation New Opportunities Grant and a grant from the British Columbia Knowledge Development Fund to TMS, and by Discovery Grants from the Natural Sciences and Engineering Research Council of Canada to TMS and VDL.

\section{References}

Chun, M. M., \& Potter, M. C. (1995). A two-stage model for multiple target detection in rapid serial visual presentation. Journal of Experimental Psychology: Human Perception and Performance, 21, 109-127.

Dell'Acqua, R., Jolicœur, P., Luria, R., \& Pluchino, P. (2009). Reevaluating encoding-capacity limitations as a cause of the attentional blink. Journal of Experimental Psychology: Human Perception and Performance, 35, 338-351.

Di Lollo, V., Kawahara, J., Ghorashi, S. M. S., \& Enns, J. T. (2005). The attentional blink: Resource depletion or temporary loss of control? Psychological Research/Psychologische Forschung, 69, 191-200.

Dux, P. E., Asplund, C. L., \& Marois, R. (2008). An attentional blink for sequentially presented targets: Evidence in favor of resource depletion accounts. Psychonomic Bulletin \& Review, 15, 809-813.

Dux, P. E., \& Marois, R. (2009). The attentional blink: A review of data and theory. Attention, Perception, \& Psychophysics, 71, 1683-1700.

Giesbrecht, B. L., \& Di Lollo, V. (1998). Beyond the attentional blink: Visual masking by object substitution. Journal of Experimental Psychology: Human Perception and Performance, 24, 1454-1466.

Jolicœur, P., \& Dell'Acqua, R. (1998). The demonstration of shortterm consolidation. Cognitive Psychology, 36, 138-202.

Kawahara, J., Di Lollo, V., \& Enns, J. T. (2001). Attentional requirements in visual detection and identification: Evidence from the attentional blink. Journal of Experimental Psychology: Human Perception and Performance, 27, 969-984. 
Kawahara, J., Zuvic, S. M., Enns, J. T., \& Di Lollo, V. (2003). Task switching mediates the attentional blink even without backward masking. Perception \& Psychophysics, 65, 339-351.

Martens, S., \& Wyble, B. (2010). The attentional blink: Past, present, and future of a blind spot in perceptual awareness. Neuroscience and Biobehavioral Reviews, 34, 947-957.

Norman, D. A., \& Bobrow, D. G. (1975). On data-limited and sourcelimited processes. Cognitive Psychology, 7, 44-64.

Olivers, C. N. L., Hulleman, J., Spalek, T., Kawahara, J., \& Di Lollo, V. (2010). The sparing is far from spurious: Reevaluating withintrial contingency effects in the attentional blink. Journal of Experimental Psychology: Human Perception and Performance. doi:10.1037/a0020379.

Olivers, C. N. L., \& Meeter, M. (2008). A boost and bounce theory of temporal attention. Psychological Review, 115, 836-863.

Olivers, C. N. L., Spalek, T. M., Kawahara, J., \& Di Lollo, V. (2009). The attentional blink: Increasing target salience provides no evidence for resource depletion. A commentary on Dux, Asplund, and Marois (2008). Psychonomic Bulletin \& Review, 16, 214-218.
Raymond, J. E., Shapiro, K. L., \& Arnell, K. M. (1992). Temporary suppression of visual processing in an RSVP task: An attentional blink? Journal of Experimental Psychology: Human Perception and Performance, 18, 849-860.

Taylor, M. M., \& Creelman, C. D. (1967). PEST: Efficient estimates on probability functions. The Journal of the Acoustical Society of America, 41, 782-787.

Visser, T. A. W. (2007). Masking T1 difficulty: Processing time and the attentional blink. Journal of Experimental Psychology: Human Perception and Performance, 33, 285-297.

Visser, T. A. W., Bischof, W. F., \& Di Lollo, V. (2004). Rapid serial visual distraction: Task-irrelevant items can produce an attentional blink. Perception \& Psychophysics, 66, 14181432.

Vogel, E. K., \& Luck, S. J. (2002). Delayed working memory consolidation during the attentional blink. Psychonomic Bulletin \& Review, 9, 739-743.

Wald, A. (1947). Sequential analysis. N.Y.: John Wiley \& Sons, Inc. 